\title{
A study on the Rhythm Pattern of klaxon sound
}

\author{
Bong-Young Kim ${ }^{1}$, Zhixing Tian² and Myung-Jin Bae ${ }^{3 *}$ \\ ${ }^{1}$ Soong-sil University, Department of Information and telecommunication Engineering, Seoul, 06978, Korea. \\ Orcid Id : 0000-0002-3553-039X \\ ${ }^{2}$ Soong-sil University, Department of Information and telecommunication Engineering, Seoul, 06978, Korea. \\ Orcid Id : 0000-0003-3882-2459 \\ ${ }^{3}$ Soong-sil University, Department of Information and telecommunication Engineering, Seoul, 06978, Korea. \\ Orcid Id : 0000-0002-7585-0400
}

\begin{abstract}
:
In order to solve the problem that the existing klaxon sound easily induces retaliation, a rhythm klaxon sound is proposed. But to a certain extent, it has the disadvantage of insufficient warning. To make up for this shortcoming, it is important to study the rhythm pattern of klaxon sound. The purpose of this paper is to research the relationship between the rhythm pattern of klaxon sound and people's psychological feelings, and find an efficient klaxon sound that can effectively prevent retaliation and has sufficient warning effect. By analyzing the brainwaves of the three different rhythmic klaxon sounds and the existing klaxon sounds, we can find the differences of brainwaves in different rhythmic modes, and objectively reflects the impact of different rhythm patterns on human psychological activities. In the MOS Test, the results of the survey subjectively reflect which rhythm pattern of klaxon sound that people prefer. In the end, it was found that the repetitive rhythm klaxon sound, which keeps the fast pace unchanged, can better prevent retaliation and a good warning effect.
\end{abstract}

Keyword: klaxon sound, rhythm pattern, retaliation, brainwaves, MOS Test

\section{INTRODUCTION}

The klaxon is a significant part of the car. While the car is driving, it can warn pedestrians of dangers and prevent traffic accidents. But it is also one of the main sources of urban noise. Despite the increasing number of revenge driving incidents caused by existing klaxon sound, they have not been well resolved. Therefore, in the previous research of the sound engineering laboratory, a rhythmic klaxon sound was proposed, and it was found that this rhythmic klaxon sound can well solve the problems that the existing horn sound brings unpleasantness to people and causes revenge driving. But there is also a problem of insufficient warning effect to a certain extent. Studies have found that changing the rhythm pattern of the klaxon sound can change the alert effect and pressure of the klaxon sound on people. So it is necessary to study the rhythm pattern of klaxon sound. This paper focus on what kind of rhythm pattern can improve the warning effect and bring less psychological pressure. The purpose of this paper is to find a rhythm pattern that will minimize the discomfort and stress caused under the case that get enough warning effect [1] [2] [3].

In the second chapter of this paper, three types of klaxon sounds are proposed. In Chapter 3, a comparative analysis is made using the brain wave analysis method. In chapter 4 , MOS TEST of these three rhythm klaxon sounds and existing klaxon sound. In chapter 5, conclusion.

\section{THREE TYPE OF RHYTHM KLAXON SOUND}

Rhythmic klaxon sound is a new type of klaxon sound that changes its working time based on the existing klaxon to create a rhythm pattern. In order to study the effect of different rhythm patterns of klaxon sounds on alertness and psychological stress, three klaxon sounds with different rhythm patterns were proposed. First, the sound rhythm gradually slows down. Second, the sound rhythm gradually becomes faster. Third, there is no change in sound rhythm. The sound amplitude of each segment of waveforms remains unchanged in the three rhythm klaxon sounds, and the time interval between the two waveforms is used as the only variable [4] [5] [6].

\subsection{Increase rhythm klaxon sound}

Press the handle switch, klaxon starts to emit the first sound wave, after a period of short time, it starts emitting the second sound wave, and then after a period of short time again, it emits the third sound wave and more. Starting from the first sound wave, the interval between each sound wave becomes smaller and smaller as time passes. In the sense of hearing, the rhythm of sound is getting faster and faster. So this kind of klaxon sound can be called increase rhythm klaxon sound. As shown in Figure 1. 


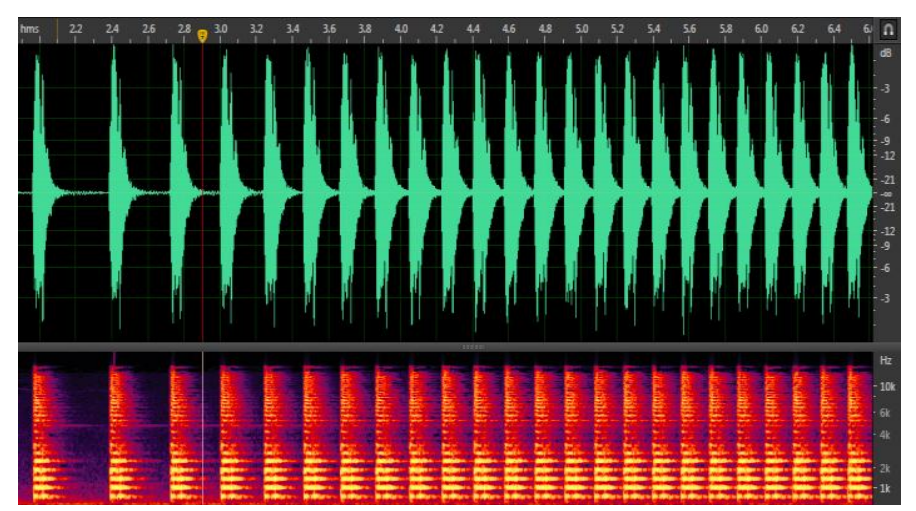

Fig 1. Waveform of increase rhythm klaxon sound

\subsection{Reduce rhythm klaxon sound}

At the beginning, each sound wave is very close together, the time interval is short, and then the time interval between sound waves gradually increases. The means that rhythm of the sound gradually decreases from fast to slow. The sound waveform of the reduce rhythm klaxon sound is shown in Figure 2.

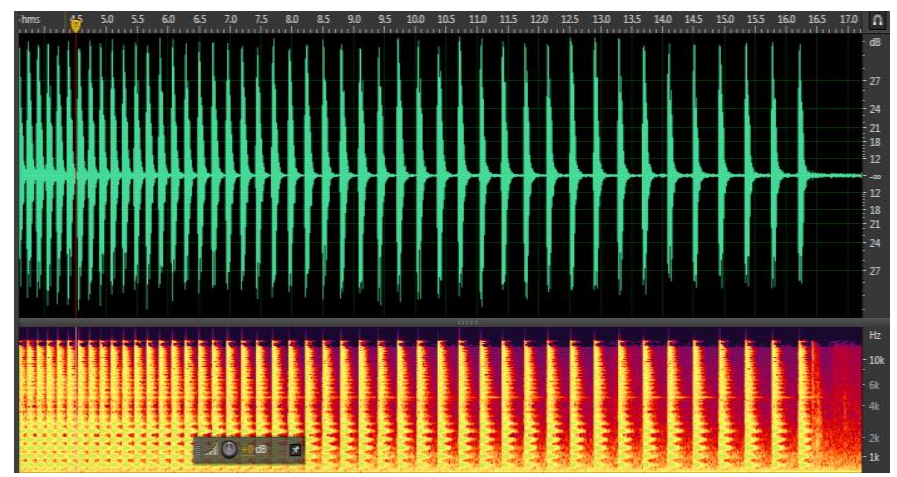

Fig 2. Waveform of the reduce rhythm klaxon sound

\subsection{Repetitive rhythm klaxon sound}

The time interval for each sound wave is exactly the same. The generation and disappearance of sound waves change periodically. So the rhythm of the sound has not changed. The waveform of the repetitive rhythm klaxon sound is shown in Figure 3.

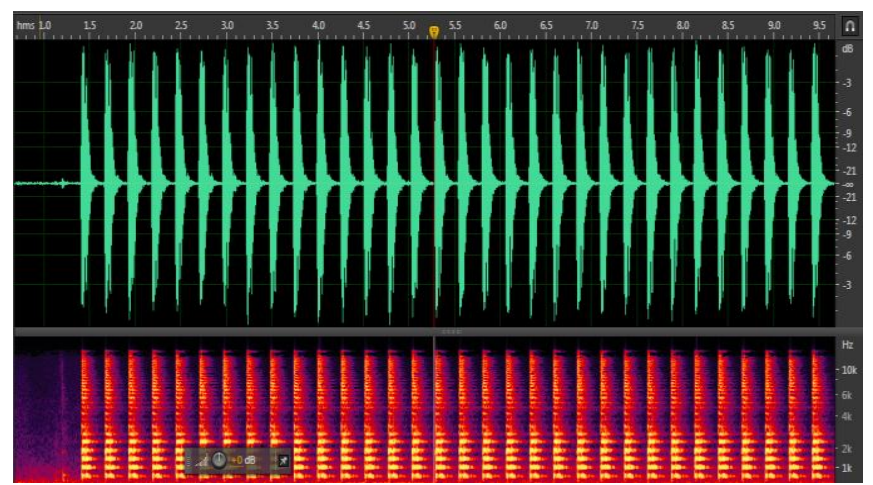

Fig 3. Waveform of the repetitive rhythm klaxon sound

\section{BRAIN WAVE ANALYSIS}

Brain waves were measured and analyzed for all there three rhythmic patterns and non-rhythmic patterns. The test environment was selected in a quiet conference room. The tester was a 20-year-old man. The klaxon was placed 3 meters away from the test subject, and then output different klaxon sounds in turn with each sound being output for one minute. After listening to a kind of klaxon sound, the tester was allowed to rest for two minutes. finally, the brain waves of the tester were measured in four different sound environments [7] [8] [9].
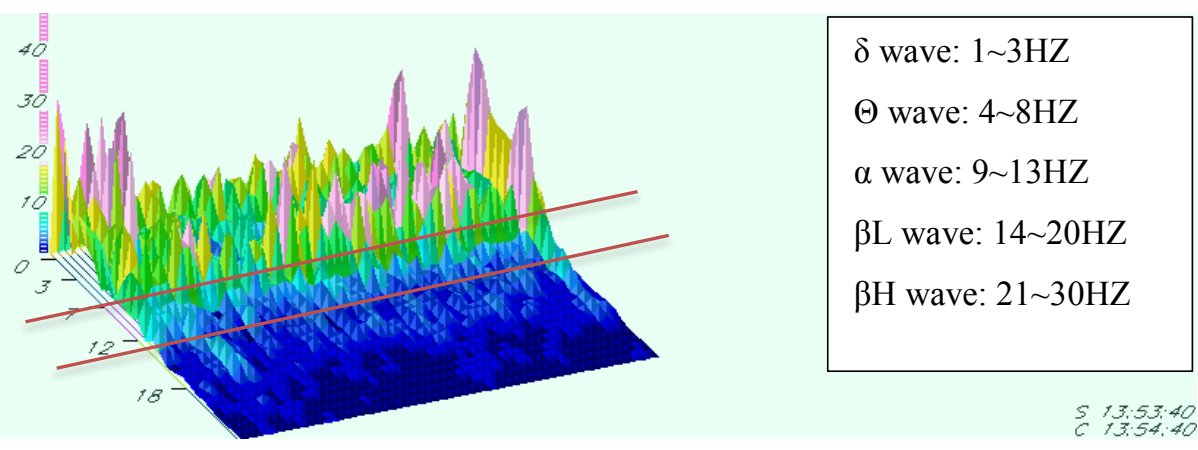

Fig 4. Brain waves with repeat rhythm klaxon sound 

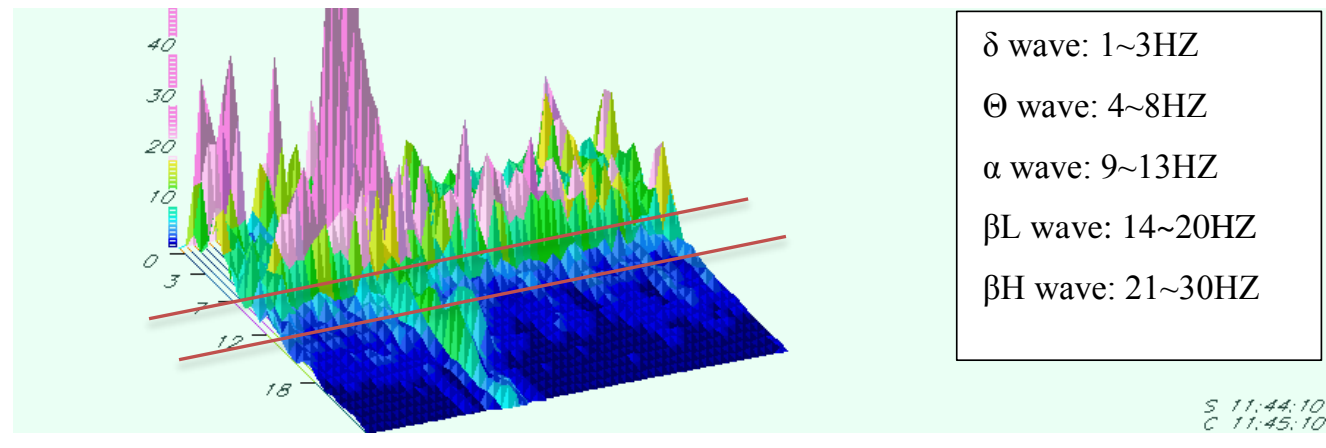

Fig 5. Brain waves with increase rhythm klaxon sound
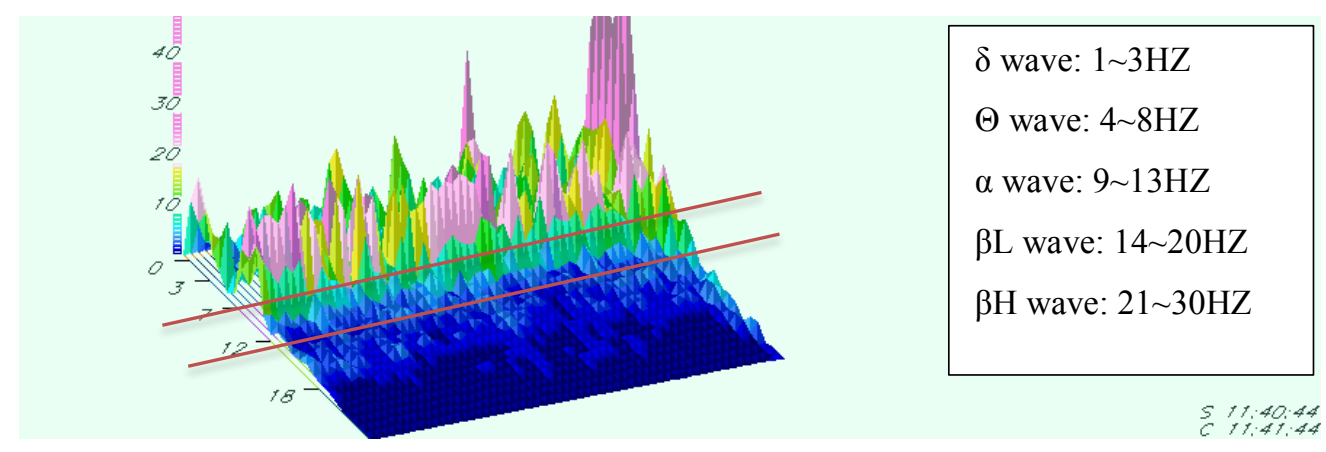

Fig 6. Brain waves with existing klaxon sound
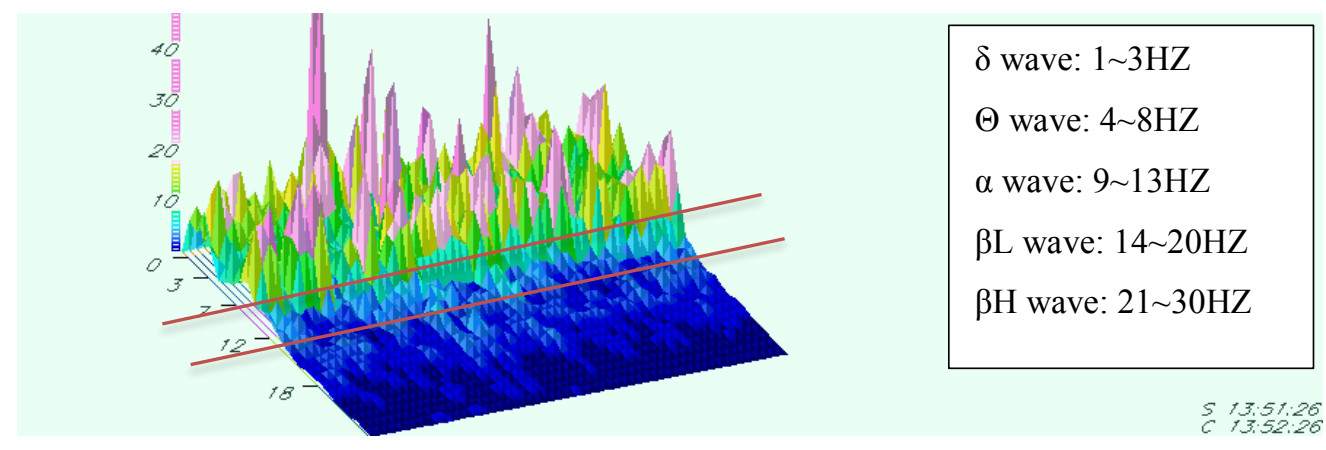

Fig 7. Brain waves with reduce rhythm klaxon sound

Figures four, five, six, and seven are the brainwave diagrams of the four types of klaxon sound. For $\delta$ and $\Theta$ waves, the existing klaxon sound is similar to reduce rhythm klaxon sound, repeat rhythm klaxon sound and increase the rhythm klaxon is almost the same, and the brain waves of the first two types of klaxon sound are more than the last two types of klaxon sound. Compared with other rhythmic klaxon sounds, the rhythmic klaxon sound has more $\alpha$ waves. As case of $\beta \mathrm{L}$ and $\beta \mathrm{H}$, repeating the rhythm, reduce the rhythm, the existing klaxon sound all generate a lot of $\beta$ waves. In contrast, reducing rhythm klaxon sound produces fewer $\beta$ waves. The comparison results of various brain waves are shown in Table. ( $\mathbf{A}$ : many, ن : much, $\mathbf{\nabla}$ : little, そ: few ) [10] [11] [12].

In brain wave analysis, the fewer $\delta, \Theta$, and $\beta$ waves and the more $\alpha$ waves, the less likely klaxon sound is to induce revenge. The more $\alpha$ and $\beta$ waves, the better the warning effect of klaxon sound. After comparing and analyzing the values of various brain waves, we can get the rankings of various klaxon sounds on the prevention of psychological retaliation and warning effects. The ranking of the four klaxon sounds is showed in table 2 [13]. 
Table 1. Ranking of various klaxon sounds

\begin{tabular}{|c|c|c|c|c|}
\hline & $\delta$ wave & $\Theta$ wave & $\alpha$ wave & $\beta \mathrm{L}, \beta \mathrm{H}$ wave \\
\hline $\begin{array}{l}\text { Repeat rhythm } \\
\text { klaxon sound }\end{array}$ & & & & \\
\hline $\begin{array}{l}\text { Increase rhythm } \\
\text { klaxon sound }\end{array}$ & & & & \\
\hline $\begin{array}{l}\text { Existing klaxon } \\
\text { sound }\end{array}$ & & & & \\
\hline $\begin{array}{l}\text { Reduce rhythm } \\
\text { klaxon sound }\end{array}$ & & & & \\
\hline
\end{tabular}

Table 2. Ranking of various klaxon sounds

\begin{tabular}{|c|c|c|}
\hline Increase rhythm klaxon sound & $\begin{array}{c}\text { Prevent retaliation } \\
(\delta, \Theta, \beta \uparrow ; \alpha \downarrow)\end{array}$ & $\begin{array}{c}\text { Warning effect } \\
(\boldsymbol{\beta}, \boldsymbol{\alpha} \uparrow)\end{array}$ \\
\hline Existing klaxon sound & $(1)$ & (4) \\
\hline Reduce rhythm klaxon sound & (4) \\
\hline Repeat rhythm klaxon sound & $(3)$ & (1) \\
\hline
\end{tabular}

\section{MOS-TEST}

In order to objectively reflect the true evaluation results of the pedestrians on the four klaxon sounds, MOS-Test was performed on the pedestrians. The test subjects were 45 college students aged 20-24. The test site is in an empty outdoor parking lot. The tester was located 3 meters in front of the car, listened to four kinds of klaxon sound, each sound for 20 seconds, and then selected his favorite klaxon sound from the four horn sounds.

The survey results showed that 3 out of 45 testers thought the existing klaxon sound was better. In addition to the two of them, 42 of them chose their own preferred rhythm of klaxon sound. The results of the survey are shown in Table 3. More than half of the testers preferred repetitive rhythm klaxon sound. There were 14 testers who thought that the reduction of rhythm was good, accounting for $33.3 \%$ of the total number. Six people preferred to increase rhythm klaxon sound, which only accounted for $14.3 \%$ of the total number. So many people think that repeat rhythm klaxon sound is better.

Table 3. People's prefer rhythm patterns

\begin{tabular}{|c|c|c|c|}
\hline & $\begin{array}{c}\text { Increase rhythm } \\
\text { klaxon sound }\end{array}$ & $\begin{array}{c}\text { Reduce rhythm } \\
\text { klaxon sound }\end{array}$ & $\begin{array}{c}\text { Repeat rhythm } \\
\text { klaxon sound }\end{array}$ \\
\hline Number of people & 6 & 14 & 22 \\
\hline percentage & $14.3 \%$ & $33.3 \%$ & $52.4 \%$ \\
\hline
\end{tabular}




\section{CONCLUSION}

The problem that the existing klaxon easily induces revenge can be solved by giving klaxon sound a rhythm pattern. By studying the relationship between the rhythm pattern of klaxon sound and people's psychoacoustics. we can find more efficient rhythm klaxon sound. An analysis of the brain waves of the three proposed rhythmic klaxon sounds and existing klaxon sounds shows that the existing klaxon sound can induce the brain to produce more $\delta, \Theta, \beta$ waves, and suppress $\alpha$ waves. Existing klaxon sound most likely to induce revenge. Increase rhythm klaxon sound produces fewer $\alpha$ and $\beta$ waves, so the warning effect is insufficient. Repeat rhythm klaxon sound is better at preventing retaliation than reducing rhythm klaxon sound. In contrast, reduce rhythm klaxon sound's warning effect is better than repeat rhythm klaxon sound.

MOS-TEST objectively reflects people's preference for some kind of klaxon sound. The survey results show that many people prefer repeated klaxon sound, followed by decrease rhythm klaxon sound and increase rhythm klaxon sound, which is consistent with the results of brain wave analysis. Repeat rhythm klaxon sound not only can effectively prevent retaliation, but also has a good warning effect.

This paper studies the influence of the changing trend of rhythm on people's psychological feelings, and concludes that repeating rhythm patterns are better. In subsequent studies, we will continue to explore the relationship between the speed of klaxon sound's repetitive rhythm and people's psychological feelings.

\section{REFERENCE}

[1] Michael S. Wogalter, Rachelle N. Ornan, Raymond W. Lim1, M. Ryan Chipley" On the Risk of Quiet Vehicles to Pedestrians and Drivers". October 1, 2001 Review Article.

[2] Zhixing Tian, Bong-Young Kim, and Myung-Jin Bae. "A study on the Improvement of Klaxon Sound". International Journal of Engineering Research and Technology. ISSN 0974-3154, Volume 12, Number 12 (2019), pp. 2439-2444

[3] Bong-Young Kim, Zhixing Tian and Myung-Jin Bae.” A Study on the Causes of Revenge Psychology of Klaxon sound" International Journal of Engineering Research and
Technology. ISSN 0974-3154, Volume 12, Number 12 (2019), pp. 2957-24962.

[4] Sathio, B., Brohi, K.M., Laghari, R. "To analyze the effect of road traffic noise on surrounding buildings".AIP Conference Proceedings 2119,020012.

[5] Vishnubhatla, A. "A new method for pedestrian alerts". International Journal of Engineering Research and Technology 11(11), pp. 1661-1683.

[6] Ahn, I.-S., Bae, M.-J.” A study on the sound warning system for prevention of roadkill accident".International Journal of Engineering Research and Technology11(10), pp. $1605-1618$.

[7] Lemaitre, Guillaume; Susini, Patrick; Winsberg, Suzanne; McAdams, Stephen; Letinturier, Boris. "The sound quality of car horns: A psychoacoustical study of timbre". Acta Acustica united with Acustica, Volume 93, Number 3, May/June 2007, pp. 457-468.

[8] Lemaitre, Guillaume; Susini, Patrick; Winsberg, Suzanne; McAdams, Stephen; Letinturier, Boris. "The Sound Quality of Car Horns: Designing New Representative Sounds" Acta Acustica united with Acustica, Volume 95, Number 2, March/April 2009, pp. 356-372.

[9] Kim, Yeon Soo; Shin, Taejin; Lee, Sang Kwon; Kim, Dae Hyun. "Acoustics Acoustic Characteristic of Car Horn Sound". Institute of Noise Control Engineering.

[10] Takada, Masayuki; Takeno, Ayako; Iwamiya, Shin-ichiro "Effects of vehicle horn acoustic properties on auditory impressions and the interpretation of reasons for horn use by other drivers" Noise Control Engineering Journal, Volume 58, Number 3, 1 May 2010, pp. 259-272.

[11] Fastl, H. "The Psychoacoustics of Sound-Quality Evaluation". Acta Acustica united with Acustica, Volume 83, Number 5, September/October 1997, pp. 754-764.

[12] Seong-Geon Bae and Myung-Jin Bae."Analysis of Health-healing Effects According to Acoustic Signal Characteristics in psychoacoustic". International Journal of Engineering Research and Technology. ISSN 09743154, Volume 12, Number 11 (2019), pp. 2057-2060.

[13] Ahn, I.-S., Kim, B.-Y., You, K.-B., Bae, M.-J. “A Study on the Characteristics of an EEG Based on a Singing Bowl's Sound Frequency". Studies in Computational Intelligence 789, pp. 233-243. 